\title{
Molecular Dynamics Simulation Studies of $p$-Xylene in OH-free Si-MCM-41
}

\author{
Tanin Nanok ${ }^{\mathrm{a}, \mathrm{b}}$, Philippe A. Bopp ${ }^{\mathrm{b}}$, and Jumras Limtrakul ${ }^{\mathrm{a}}$ \\ ${ }^{a}$ Laboratory for Computational and Applied Chemistry (LCAC), Physical Chemistry Division, \\ Faculty of Sciences, Kasetsart University, Bangkok 10900, Thailand \\ b Laboratoire de Physico-Chimie Moléculaire (LPCM UMR 5803), Université Bordeaux 1, \\ 351 Cours de la Libération, F-33405 Talence CEDEX, France
}

Reprint requests to Prof. P. A. B.; E-mail: pab@loriot.lsmc.u-bordeaux.fr

Z. Naturforsch. 60a, $805-813$ (2005); received September 2, 2005

We report on modeling efforts and molecular dynamics computer simulations of the structure and self-diffusion of $p$-xylene in OH-free Si-MCM-41 as a function of loading. Both the guest molecules and Si-MCM-41 are modeled as flexible entities. With this newly developped intermolecular force field the average potential energy of $p$-xylene in the pore increases with increasing loading. The adsorption of $p$-xylene in MCM-41 is primarily associated with the van der Waals interactions of the model, whereas the contribution from electrostatic interactions is relatively small (about $2 \mathrm{kcal} / \mathrm{mol}$ ), in accordance with other aromatic hydrocarbons adsorbed in zeolite catalysts. The calculated selfdiffusion coefficients of $p$-xylene in Si-MCM-41 are well comparable with diffusion coefficients of pyridine in MCM-41 and of the same order of magnitude as in liquid $p$-xylene. Increasing the loading results in non-negligible mutual $p$-xylene interaction, thus leading to a decrease of the self-diffusion coefficient.

Key words: Molecular Dynamics; Adsorption; Diffusion; p-Xylene; Si-MCM-41.

\section{Introduction}

MCM-41 is a novel mesoporous material which has a large surface area and adsorption capacity [1-3]. It can be synthesized in its high-silica form (Si-MCM41) and also in its high-alumina form (Al-MCM41) $[4,5]$. The high-silica form of MCM-41 is structurally stable to thermal and hydrothermal treatment. It has, therefore, great potential for practical use as an adsorbent and mesoporous support for depositing active catalysts [6]. Unlike zeolites, its large pore diameter with fewer diffusional constraints can prevent coke formation which can build up due to bulky products not being able to diffuse out. In addition, this material also permits the simultaneous entry of several reactant molecules into the pores [7,8]. Although the reactivity of MCM-41 has been extensively investigated experimentally [9], relatively few theoretical studies have been reported [10-16]. This might be due to the large number of atoms necessary to describe the MCM-41 structure without applying restrictive symmetry constraints. Furthermore, complete structural information at the atomic level is still not available.
Research on adsorption and diffusion of aromatics in MCM-41 is of particular interest since many separation processes and reactions of industrial interest in MCM-41 are concerned with bulky aromatic compounds [17]. However, only a few adsorption isotherms of aromatic hydrocarbons in MCM-41 have been determined experimentally. Benzene, toluene, $p$-xylene, mesitylene, and naphthalene have nevertheless been investigated in a wide range of temperatures [2,1820]. Understanding the adsorption and diffusion mechanisms will be helpful for the interpretation of other reactions in this material. These phenomena can be observed experimentally by a variety of methods, mostly spectroscopic, which are, however, often particularly difficult in porous materials. Furthermore, it is usually not possible to derive a consistent microscopic picture of the diffusion process, or processes, only from the experiments.

Atomistic molecular dynamics (MD) simulations are thus a choice technique to supplement experiments since they provide a full description of the atomic motions and the microstructure that give rise to the observed properties. This approach has been shown to be effective in exploring the microscopic mobility and dif- 


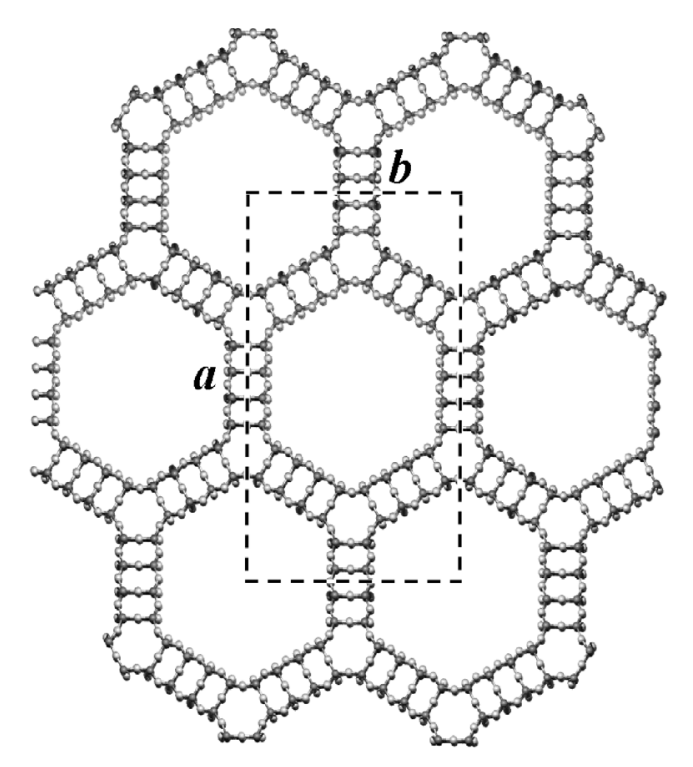

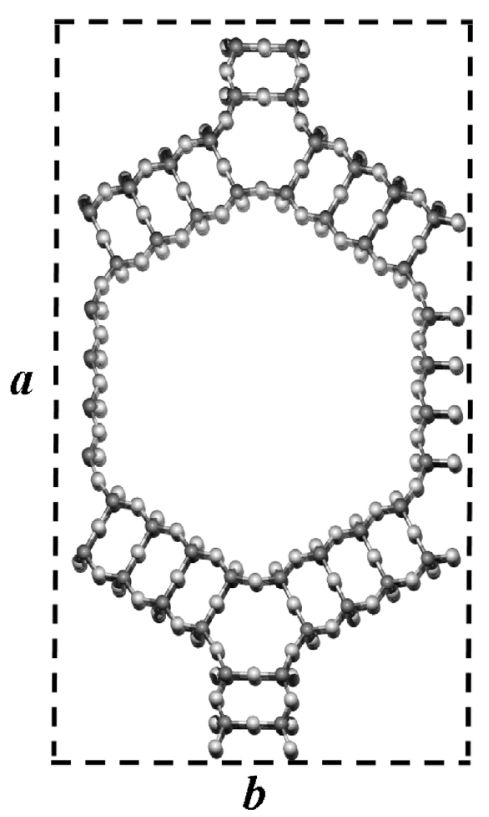

Fig. 1. Model of SiMCM-41; the hexagonal lattice framework (left) and the Si-MCM-41 supercell (right). fusion of guest molecules in porous materials [21]. To gain insight into the adsorption and diffusion mechanism of aromatic hydrocarbons in mesoporous materials is the aim of our present study.

The structure of MCM-41 was modeled in its highsilica form with $p$-xylene serving as a prototype aromatic molecule diffusing in the pores. This being a preliminary study, the silanol groups are not explicitely taken into account. Their content in the channel can be estimated to be about one $\mathrm{OH}$ group per $20 \mathrm{SiO}_{2}$ units [22]. While the specifics of the adsorption process will be affected by these groups, the average structural and diffusional properties should be much less influenced, especially at higher loadings. The present study will serve as a reference for comparison with ongoing work including these groups in the model.

\section{Methodology}

\subsection{Construction of the Si-MCM-41 Structure}

From high resolution electron microscopy measurements, it was concluded that the pores of MCM-41 have essentially one-dimensional cylinder-like shapes and are arranged parallel to each other in a honeycombtype lattice, without intersections. These materials can be prepared with pore diameters ranging from 15 to $100 \AA[1,2]$. Interestingly, both circular and hexagonal cross sections have been visualized $[23,24]$. Al- though the structure of MCM-41 has been extensively studied [1-3], detailed crystallographic data at the atomic level are still lacking. In this study, the highsilica form of amorphous MCM-41 was modeled as a hypothetic Si-MCM-41 perfect lattice. The initial atomic coordinates were taken from the work of $\mathrm{Gu}-$ sev [25] and partially modified in order to achieve the periodic framework needed for the simulation. The simulations were performed on an orthorhombic supercell $(a=39.37 \AA, b=22.73 \AA, c=45.99 \AA ; \alpha=$ $\beta=\gamma=90^{\circ}$ ) comprising 1440 atoms (480 Si-atoms and $960 \mathrm{O}$-atoms). Each supercell contained two parallel hexagonal channels with a diameter of about $20 \AA$, running along the $c$-axis, as illustrated in Figure 1.

\subsection{Model and Potential Parameters}

It is known that when guest molecules diffuse inside the pores of certain zeolites, the conformation of the zeolite is modified to a certain extent in order to achieve the best energetic and shape complementarity with the guest molecules. In some cases, diffusion is not possible at all without a concomitant deformation of the lattice. Furthermore, especially at lower loadings, the thermalization of guest molecules can be enhanced by the lattice framework [26]. Thus, and also for consistency reasons with ongoing work on smallpore zeolites [27], both the MCM-41 framework and $p$-xylene were modeled as flexible entities in order to 
mimic these features of the real systems as closely as possible.

The interaction model for the lattice, a generalized valence force field (GVFF), is taken from the work of Nicholas and coworkers [28]. In this approximation, the potential energy of the framework system is represented by $\mathrm{Si}-\mathrm{O}$ bond stretches as well as $\mathrm{O}-\mathrm{Si}-\mathrm{O}$ and $\mathrm{Si}-\mathrm{O}-\mathrm{Si}$ angle bends. The bond stretches and $\mathrm{O}-\mathrm{Si}-\mathrm{O}$ angle bends were approximated as simple harmonic potentials, while the Si-O-Si bond bends, which are highly anharmonic (and the equilibrium values of which are known to vary for different silicates) were modeled with a quartic potential. The equilibrium bond lengths and bond angles were derived from structural data [25]. In modeling the framework flexibility of our hypothetic Si-MCM-41, we were guided by the experimental vibrational spectra. We note, however, that the low frequency side of the spectrum is distorted by the finite box size of our simulated system, see Section 2.4. It has been shown that the inclusion of additional non-bonded long range or cross terms does little to improve the structure and the quality of the vibrational spectra $[28,29]$. We took advantage of this by not including such terms in our calculations. The potential energy of the zeolite framework can be written as sums of terms:

$$
\begin{aligned}
V_{\text {MCM-41 }}= & \sum\left(\frac{k_{r}}{2}\left(r-r_{0}\right)^{2}\right) \\
+ & \sum\left(\frac{k_{\theta}}{2}\left(\theta-\theta_{0}\right)^{2}\right) \\
+ & \sum V_{\text {bend }}(\mathrm{Si}-\mathrm{O}-\mathrm{Si}) \\
V_{\text {bend }}(\mathrm{Si}-\mathrm{O}-\mathrm{Si})= & \frac{k_{\theta}^{\prime}}{2}\left(\theta-\theta_{0}\right)^{2}+\frac{k_{\theta}^{\prime \prime}}{3}\left(\theta-\theta_{0}\right)^{3} \\
& +\frac{k_{\theta}^{\prime \prime \prime}}{4}\left(\theta-\theta_{0}\right)^{4},
\end{aligned}
$$

where the sums are taken over all coordinates of a given type; $k_{r}$ is the force constant of the $\mathrm{Si}-\mathrm{O}$ bond stretch, $k_{\theta}$ is the force constant for the O-Si-O angle bend, $k_{\theta}^{\prime}, k_{\theta}^{\prime \prime}$, and $k_{\theta}^{\prime \prime \prime}$ are the force constants for the $\mathrm{Si}-\mathrm{O}-\mathrm{Si}$ angle bend, respectively. $r$ and $\theta$ are the actual values of bond distances and bond angles, and the zero index denotes their equilibrium values. The force field parameter values for Si-MCM-41 are listed in Table 1.

The standard force field (3) developed by P. A. Kollman et al. [30] (AMBER) was employed to evaluate the intramolecular potential for $p$-xylene. Four different atoms are defined for $p$-xylene: CA, CT, HA,
Table 1. The potential parameters for the Si-MCM-41 lattice, see (1) and (2).

\begin{tabular}{lll}
\hline Internal coordinate & Equilibrium values & Force constants \\
\hline Si-O stretch & $r_{0}=1.591 \AA$ & $k_{r}=597.32^{\mathrm{a}}$ \\
O-Si-O angle bend & $\theta_{0}=109.5^{\circ}$ & $k_{\theta}=138.12^{\mathrm{b}}$ \\
$\mathrm{Si}-\mathrm{O}-\mathrm{Si}$ angle bend & $\theta_{0}=155.0^{\circ}$ & $k_{\theta}^{\prime}=10.85^{\mathrm{b}}$ \\
& & $k_{\theta}^{\prime \prime}=-34.08^{\mathrm{c}}$ \\
& & $k_{\theta}^{\prime \prime \prime}=26.52^{\mathrm{d}}$ \\
\hline Atomic charges & $\mathrm{Si}$ & $\mathrm{O}$ \\
\hline$|e|$ & 2.000 & -1.000 \\
\hline
\end{tabular}

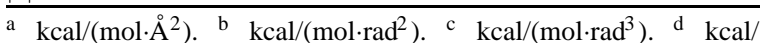
$\left(\mathrm{mol} \cdot \mathrm{rad}^{4}\right)$.

HT, the atoms labeled A belonging to the aromatic ring and those marked $\mathrm{T}$ forming part of the methyl groups. The equilibrium bond lengths and bond angles were obtained from ab initio geometry optimizations at the HF/6-31G(d,p) level. The "non-bonded interactions" were applied only for atoms separated by at least three bonds (1-4 interactions) and reduced by using factors of $1 / 1.2$ and $1 / 2$ for the $1-4$ electrostatic and van der Waals terms, respectively. The values of the point charges were estimated according to the ChelpG scheme [31] from the wave functions of isolated molecules. Thus, for one molecule

$$
\begin{aligned}
& V_{\text {xylene }} \\
& =\sum\left(\frac{k_{r}}{2}\left(r-r_{0}\right)^{2}\right)+\sum\left(\frac{k_{\theta}}{2}\left(\theta-\theta_{0}\right)^{2}\right) \\
& +\sum k[1+\cos (n \phi-\delta)] \\
& +\sum_{\text {n.b. }}\left(4 \varepsilon_{i j}\left[\left(\frac{\sigma_{i j}}{r_{i j}}\right)^{12}-\left(\frac{\sigma_{i j}}{r_{i j}}\right)^{6}\right]+c \cdot \frac{q_{i} q_{j}}{r_{i j}}\right),
\end{aligned}
$$

where the three sums are taken over the appropriate coordinates and the last one (n. b.) over all 'non-bonded' intramolecular distances.

The intermolecular guest-guest interactions and the interactions between guest molecules and MCM-41 were expressed, like the non-bonded interactions, as sums of Lennard-Jones (LJ 12-6) and Coulomb terms between atomic sites. The LJ- and charge parameters for xylene are identical in both cases:

$$
\begin{aligned}
& V_{\text {xylene-xylene }}=V_{\text {zeolite-xylene }} \\
& =\sum\left(4 \varepsilon_{i j}\left[\left(\frac{\sigma_{i j}}{r_{i j}}\right)^{12}-\left(\frac{\sigma_{i j}}{r_{i j}}\right)^{6}\right]+c \cdot \frac{q_{i} q_{j}}{r_{i j}}\right)
\end{aligned}
$$

where $r_{i j}$ is the distance between two sites $i$ on $p$ xylene and $j$ on either $p$-xylene or the lattice. $\varepsilon_{i j}$ and $\sigma_{i j}$ are energy and size parameters characteristic of the 
Table 2. Potential parameters for the non-bonding xylenexylene and xylene-MCM-41 interactions, see (4). Atom labels see text.

\begin{tabular}{cccccc}
\hline $\begin{array}{c}\text { Lennard- } \\
\text { Jones }\end{array}$ & $\begin{array}{c}\varepsilon_{i j} \\
(\mathrm{kcal} / \mathrm{mol})\end{array}$ & $\begin{array}{c}\sigma_{i j} \\
(\AA)\end{array}$ & $\begin{array}{c}\text { Lennard- } \\
\text { Jones }\end{array}$ & $\begin{array}{c}\varepsilon_{i j} \\
(\mathrm{kcal} / \mathrm{mol})\end{array}$ & $\begin{array}{c}\sigma_{i j} \\
(\AA)\end{array}$ \\
\hline CA-CA & 0.0859 & 3.40 & CA-HA & 0.0359 & 3.00 \\
HA-HA & 0.0150 & 2.60 & CT-CT & 0.1093 & 3.40 \\
CT-HT & 0.0422 & 3.02 & HT-HT & 0.0157 & 2.65 \\
CA-CT & 0.0970 & 3.40 & CA-HT & 0.0367 & 3.02 \\
HA-CT & 0.0405 & 3.00 & HA-HT & 0.0153 & 2.62 \\
CA-Si & 0.0643 & 3.57 & HA-Si & 0.0269 & 3.17 \\
CT-Si & 0.0724 & 3.57 & HT-Si & 0.0275 & 3.20 \\
CA-O & 0.1173 & 3.17 & HA-O & 0.0490 & 2.77 \\
CT-O & 0.1321 & 3.17 & HT-O & 0.0501 & 2.80 \\
\hline
\end{tabular}

Table 3. Partial charges on the atoms of $p$-xylene, see (3) and (4). Atom labels see text.

\begin{tabular}{lclclc}
\hline Atom & charge $|e|$ & Atom & charge $|e|$ & Atom & charge $|e|$ \\
\hline $\mathrm{CA}^{\mathrm{a}}$ & -0.205 & $\mathrm{CA}^{\mathrm{b}}$ & 0.180 & $\mathrm{CT}$ & -0.222 \\
$\mathrm{HA}$ & 0.130 & $\mathrm{HT}$ & 0.064 & & \\
\hline a Aromatic $\mathrm{C}$ atom connected to $\mathrm{H}$ atom. ${ }^{\mathrm{b}}$ Aromatic $\mathrm{C}$ atom con- \\
nected to $\mathrm{CH}_{3}$ group.
\end{tabular}

$i$ - $j$ interaction, see Table $2, q_{i}$ are the partial charges associated with the sites (Tables 1 and 3), and $c$ is a constant.

The LJ-parameters between $p$-xylene and the atomic framework of MCM-41 were derived by fitting only the $\varepsilon$ - and $\sigma$-values associated with the lattice atoms $\mathrm{O}$ and $\mathrm{Si}$ to the experimental heat of adsorption of $p$-xylene in Si-MCM-41 [19,32]. Half the formal charges were assigned to the $\mathrm{Si}$ and O-atoms of the Si-MCM-41 framework [33]. The complete set of potential parameters is given in Tables $1-4$.

\subsection{Molecular Dynamics Simulations}

The simulations were performed with a constant number of particles $N$ and volume $V$ and at a constant total energy $E$ ( $N V E$-ensemble) at an average temperature of $300 \mathrm{~K}$ with the DL-POLY program package [34] mainly on modern Linux workstations. Orthorhombic periodic boundary conditions were applied to include the effects of the infinite framework. Additionally to runs with only one xylene guest molecule, loadings of $16,32,48$, and 64 molecules per Si-MCM-41 supercell, corresponding to 8, 16, 24, and 32 molecules per hexagonal channel in our simulation box, or experimental loadings of roughly 0.55 , $1.11,1.66$, and $2.22 \mathrm{mmol}$ of $p$-xylene per gram of Si-MCM-41 (mmol/g), were simulated. The different loadings were modeled with the same periodic boundary condition. The electrostatic interactions were com-
Table 4. The parameters for the intramolecular potential of $p$-xylene, see (3). Atom labels see text.

\begin{tabular}{|c|c|c|c|}
\hline Bond stretch & & $k_{r}\left(\mathrm{kcal} / \mathrm{mol} \cdot \AA^{2}\right)$ & $r_{0}(\AA)$ \\
\hline$\overline{\mathrm{CA}-\mathrm{CA}}$ & & 938.00 & 1.387 \\
\hline CA-HA & & 734.00 & 1.076 \\
\hline CA-CT & & 634.00 & 1.510 \\
\hline CT-HT & & 680.00 & 1.085 \\
\hline Angle bend & & $k_{\theta}\left(\mathrm{kcal} / \mathrm{mol} \cdot \mathrm{rad}^{2}\right)$ & $\theta_{0}\left(^{\circ}\right)$ \\
\hline$\overline{\mathrm{CA}-\mathrm{CA}-\mathrm{CA}}$ & & 126.00 & 120.00 \\
\hline CA-CA-HA & & 70.00 & 120.00 \\
\hline CA-CA-CT & & 140.00 & 120.00 \\
\hline CA-CT-HT & & 100.00 & 109.50 \\
\hline HT-CT-HT & & 70.00 & 109.50 \\
\hline Dihedral & $k(\mathrm{kcal} / \mathrm{mol})$ & $n$ & $\delta$ (Degrees) \\
\hline$\overline{\mathrm{X}-\mathrm{CA}-\mathrm{CA}-\mathrm{X}}$ & 3.6250 & 2.0 & 180.0 \\
\hline X-CA-CT-X & 0.0000 & 2.0 & 0.0 \\
\hline
\end{tabular}

puted using the Ewald sum method, and the van der Waals interactions were evaluated within a cutoffradius of $11 \AA$. The Verlet algorithm was used with an integration time step of $1 \mathrm{fs}$.

The starting configurations of the mesopore-xylene systems were first equilibrated by running a simulation at $0 \mathrm{~K}$ for $50 \mathrm{ps}$ using a strongly coupled thermostat. This is a crude energy minimizer to help the system to relax before a simulation begins. The last configuration from this run was used as input for a 100 ps equilibration period at the desired temperature, $300 \mathrm{~K}$ (velocity rescaling). After this period, the velocity rescaling algorithm was removed and a run of $50 \mathrm{ps}$ was performed to ensure that there were no further drifts. After that the coordinates and velocities of the atoms were stored every $10 \mathrm{fs}$ for an additional 100 ps run for later analysis.

\subsection{Structure and Dynamics of Empty Si-MCM-41, Test of Force Field}

Before undertaking the molecular dynamics simulations of the loaded systems, the initial structure of SiMCM-41 was equilibrated for $200 \mathrm{ps}$ within the $\mathrm{NpT}$ ensemble to ensure that the selected structure (Fig. 1) does correspond to a stable minimum of our model potential, e.g., among other things, that the simulation box does not expand or contract unreasonably. The cell dimensions after this run, $a=39.26 \AA, b=22.67 \AA$, and $c=45.86 \AA$ are thus very close to the initial values of $a=39.37 \AA, b=22.73 \AA$, and $c=45.99 \AA$ for the $20 \AA$ pore of Gusev [25]. Other structural features were checked after the $N p T$ run by computing the radial pair distribution functions of the system. The last configuration of this run was used as the starting configuration for the simulations of the loaded systems. 


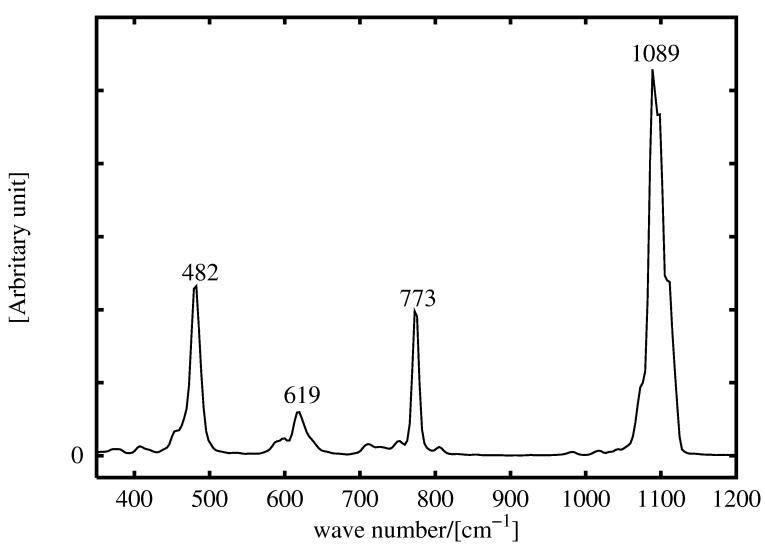

Fig. 2. Simulated IR spectrum of the model Si-MCM-41.

The infrared spectrum of Si-MCM-41 was calculated by Fourier transform of the autocorrelation function of the total dipole moment of the system, using the same magnitude of the charge values used in the Si-MCM-41 model. Experimentally, the peaks observed between 500 and $1200 \mathrm{~cm}^{-1}$ are assigned to framework vibrations [35]. The theoretical spectrum obtained from our simple model shown in Fig. 2 agrees reasonably well with the experimental data [35]. The intense peak at $1089 \mathrm{~cm}^{-1}$ is attributed to the asymmetric stretching of the Si-O bonds. The symmetric stretching modes of these bonds are predicted at $773 \mathrm{~cm}^{-1}$, and the peaks at 482 and $619 \mathrm{~cm}^{-1}$ are due to the bending modes of Si-O-Si. These spectral features resemble those reported for highly siliceous zeolites [36].

\section{Results and Discussion}

\subsection{Adsorption of p-Xylene}

The adsorption of aromatic hydrocarbons in SiMCM-41 is expected to result mainly from weak interactions of the $\pi$-electrons of the aromatic ring with the terminal silanol $(\mathrm{Si}-\mathrm{OH})$ groups [37], which are not modeled explicitly here. These interactions are included implicitly in our fitting procedure which reproduces the (average) experimental heat of adsorption. The adsorption energies reported in Table 5 are averages, i. e. the total guest-host interaction energies of the simulated system divided by the number of guest molecules. At our level of approximation, this energy can be identified with the heat of adsorption. We study its loading dependence and its decomposition into the van der Waals (vdW) and the Coulomb components,
Table 5. Simulated van der Waals- (vdW), Coulomb-, and total interaction energies and self-diffusion coefficients at $T=300 \mathrm{~K}$ and different $p$-xylene loadings.

\begin{tabular}{|c|c|c|c|c|}
\hline Loading & \multicolumn{3}{|c|}{ Energy $(\mathrm{kcal} / \mathrm{mol})$} & $\bar{D}$ \\
\hline Molecule(s)/supercell & $\mathrm{vdW}$ & Coulomb & Total & $\left(\mathrm{cm}^{2} / \mathrm{s}\right)$ \\
\hline 1 & -10.0 & -1.8 & -11.8 & - \\
\hline 16 & -10.8 & -1.9 & -12.7 & $7.8 \times 10^{-5}$ \\
\hline 32 & -11.3 & -2.0 & -13.3 & $5.3 \times 10^{-5}$ \\
\hline 48 & -11.6 & -2.0 & -13.6 & $2.8 \times 10^{-5}$ \\
\hline 64 & -11.9 & -2.0 & -13.9 & $1.8 \times 10^{-5}$ \\
\hline
\end{tabular}

as shown in Table 5. The average energies were obtained from 2600 evenly spaced configurations taken after the equilibration period. At the lowest coverage that we can study ( $N=1$ molecule per supercell), this energy is $11.8 \mathrm{kcal} / \mathrm{mol}$, in good agreement with experimental heats of adsorption of 10.4 and $12.3 \mathrm{kcal} / \mathrm{mol}$ for Si-MCM-41 and H-Al-MCM-41, respectively [19]. The former value is the one the interaction parameters were fitted to. Since $p$-xylene is a non-polar molecule, the long-range electrostatic contributions are not expected to be significant, and we find indeed that the heat of adsorption is mainly contributed from the dispersive interactions (see Table 5). A similar observation was made for the adsorption of benzene on zeolite catalysts, also yielding an electrostatic contribution of only $1-2 \mathrm{kcal} / \mathrm{mol}$ [38].

Exploring the variation of the heat of adsorption with sorbate loading, a series of $N V E$ simulations was performed on the $p$-xylene/Si-MCM-41 system, varying the number of guest molecules, $N$, as described above. Each channel was loaded with an equal number of guest molecules (except for the case of one molecule per supercell). It is found that the magnitude of adsorption energy of $p$-xylene slightly increases with increasing loading, which is similar to other hydrocarbons in narrow pore MCM-41 [39] like the one we used in this study. The loading dependence results from a balance between guest-guest and guest-host interactions. The overall trend shows that the guest-guest terms are able to overcompensate whichever less favorable guest-host interactions may arise due to mutual hindrances between guest molecules.

\subsection{Self-diffusion of $p$-Xylene}

During the simulation, configurations were saved every $10 \mathrm{fs}$. The mean square displacements (MSD) of the center of mass of the $p$-xylene molecules are plotted as a function of time for different loadings in Figure 3. The self-diffusion coefficients were then de- 


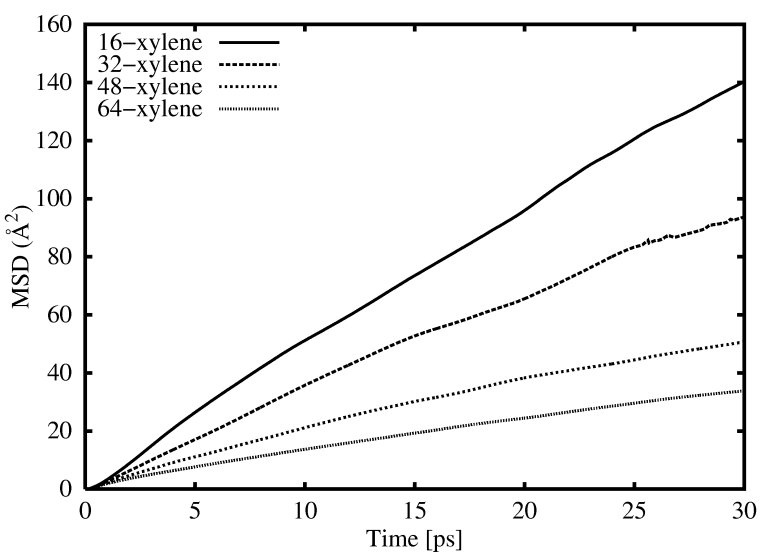

Fig. 3. Mean square displacements (MSDs), at $300 \mathrm{~K}$, of the centers of mass of the $p$-xylene molecules in Si-MCM- 41 at different loadings.

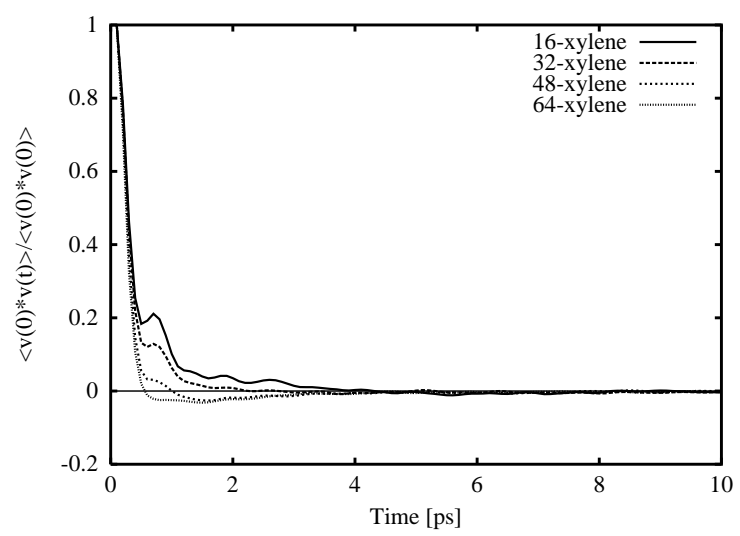

Fig. 4. The normalized autocorrelation functions, at $300 \mathrm{~K}$, of the velocity of the mass centers of the $p$-xylene molecules in Si-MCM-41 at different loadings.

termined using the Einstein relation

$$
\left\langle X^{2}(t)\right\rangle=6 D t+B
$$

where $X^{2}(t)$ is the mean square displacement at time $t$, $D$ is the self-diffusion coefficient, and $B$ is the thermal factor arising from atomic vibrations. The selfdiffusion coefficients were obtained from the slope of the curves in Figure 3. At times larger than 10 ps, the MSD is linear in time and the velocity autocorrelation functions tend to zero (Fig. 4), indicating that the diffusive regime has set in. The self-diffusions of $p$-xylene at different loadings are listed in Table 5. For the lowest loading ( $N=1$ molecule per supercell), however, the statistics were too poor to derive a meaningful value.

The calculated self-diffusion coefficients decrease as loading increases. The experimental self-diffusion

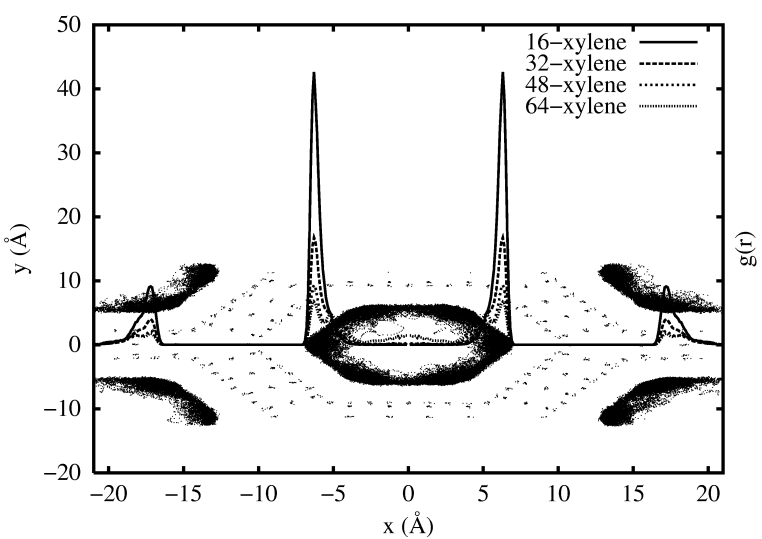

Fig. 5. Projections of the trajectories of the centers of mass of $p$-xylene (grey zone) and the framework atoms (fuzzy dots) for $100 \mathrm{ps}$ onto the $x y$-plane at a loading of 16 molecules/supercell; resulting cylindrical $g(r)$ distribution function of the $p$-xylene centers of mass with respect to the center of the channel for this and other loadings.

coefficient is not available for $p$-xylene in MCM-41. Our calculated self-diffusion coefficients are of the same order of magnitude as found in a previous MD simulation for liquid $p$-xylene at $293.15 \mathrm{~K}$ of $1.48 \cdot 10^{-5} \mathrm{~cm}^{2} / \mathrm{s}$ [40]. Comparing to another experimental system, which is related to our study, the self-diffusion coefficients obtained from our simulations are comparable with the diffusion coefficient of pyridine in MCM-41 at $298 \mathrm{~K}\left(1.0 \cdot 10^{-5}\right.$ $6.8 \cdot 10^{-5} \mathrm{~cm}^{2} / \mathrm{s}$ ) [41], even though the polarities of the two molecules are quite different.

We note that the loading dependence found here is different from the one reported for pyridine in silica MCM-41 [41]. Our $D$-values decrease by a factor of about five with increasing loading in the range studied while an increase with increasing loading is reported in [41]. The two cases are, however, not directly comparable: Besides the difference in polarity already mentioned, the geometric conditions in the two cases differ: In the MCM-41 studied here, the pore diameter is about $18-19 \AA$, while it is $33 \AA$ for silica MCM- 41 . Even at the highest loading the interaction of $p$-xylene will be dominated by the guest-wall terms in the narrower pore, and mutual steric hindrances will lower $D$. In contrast, the increased diffusivity in the wide pore is ascribed to the onset of diffusion in a second, possibly more liquid-like, layer, which fills up with increasing loading.

Figure 5 shows the radial distribution functions of the center of mass of $p$-xylene from the channel center of MCM-41. Note that the normalization of this func- 


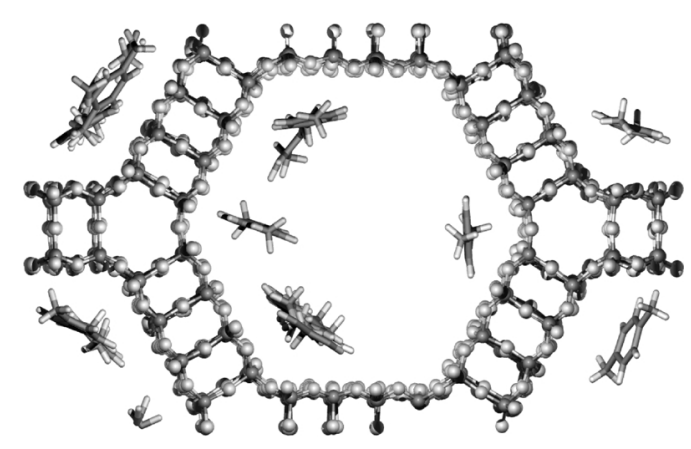

(a)

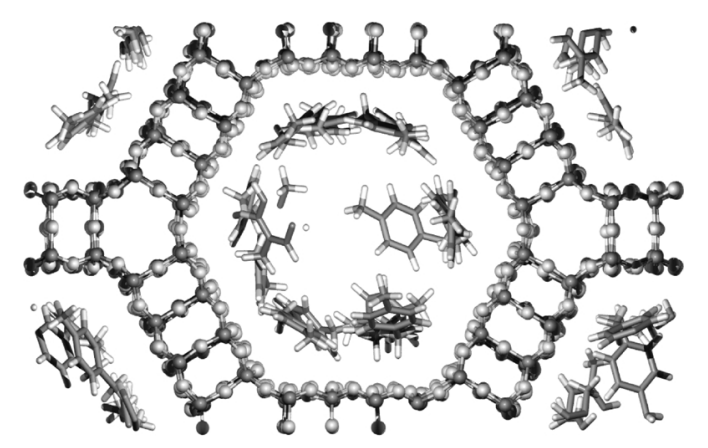

(b)

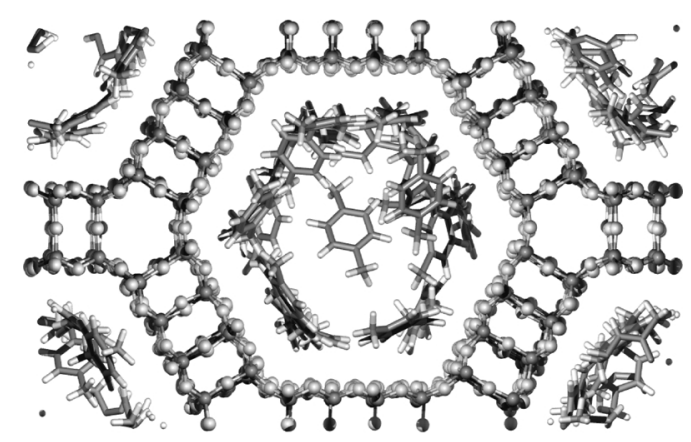

(c)

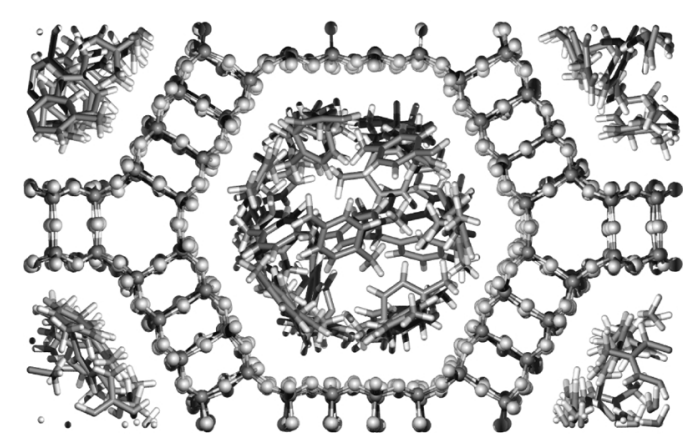

(d) $\longleftarrow$ Fig. 6. Snapshots of the distribution of $p$-xylene molecules in a Si-MCM-41 supercell at loading of (a) 16, (b) 32, (c) 48, and (d) 64 molecules/supercell after 100 ps of MD equilibrium.

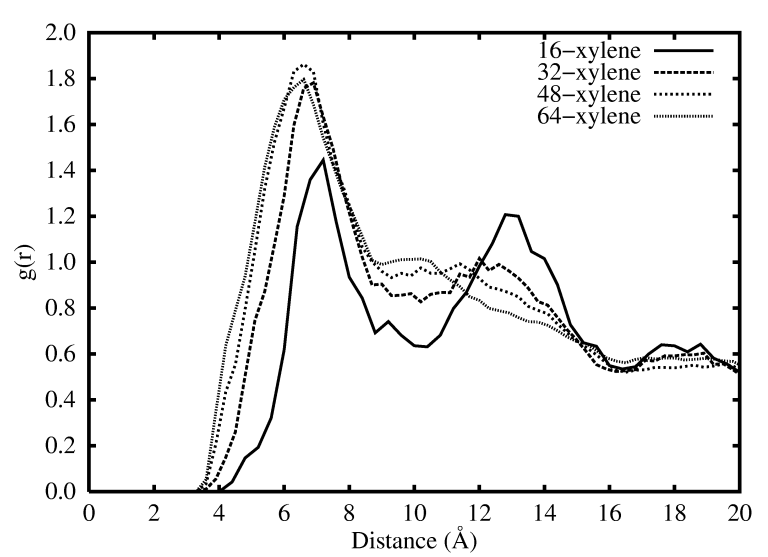

Fig. 7. The center-center pair distribution functions of $p$ xylene in Si-MCM-41 at different loadings.

tion is to the average density of $p$-xylene, the curve for the lowest loading thus has the largest amplitude. These average densities $N / V$ refer to the total volume $V$ of the simulation box, not to the volume accessible to $p$-xylene, which is difficult to determine as it can, e.g. depend on the temperature. The curves thus do not converge to 1 at large distances. The single peak found at a loading of 16 molecules/supercell at about $6 \AA$ from the center (i.e. about $3 \AA$ from the "wall") splits into two with increasing loading. The main peak moves to slightly closer distances from the wall and a second peak builds up at about $4 \AA$ from the walls. The first layer $p$-xylene molecules are thus increasingly oriented "parallel" to the wall, as seen in the snapshots of Figs. $6 a-d$.

The center of mass pair distribution functions of $p$ xylene in MCM-41 at different loadings are shown in Figure 7 . We note that while the peaks broaden with increasing loading, the first maximum peak of the centercenter pair distribution functions of $p$-xylene moves to slightly shorter distances, to the value of about $6 \AA$. This value is close to the intermolecular separation in a $p$-xylene adduct, derived at the B3LYP/6-31G(d,p) level of theory, of $5.8 \AA$ (see Fig. 8). This type of configuration can be observed frequently during the diffusion of $p$-xylene in the pores (see Fig. 8). The shortest intermolecular distance during the diffusion of $p$ xylene in the pore is about $3.6 \AA$, which is the distance between two $p$-xylene molecules in stacked configuration. The probability of distances between this value 

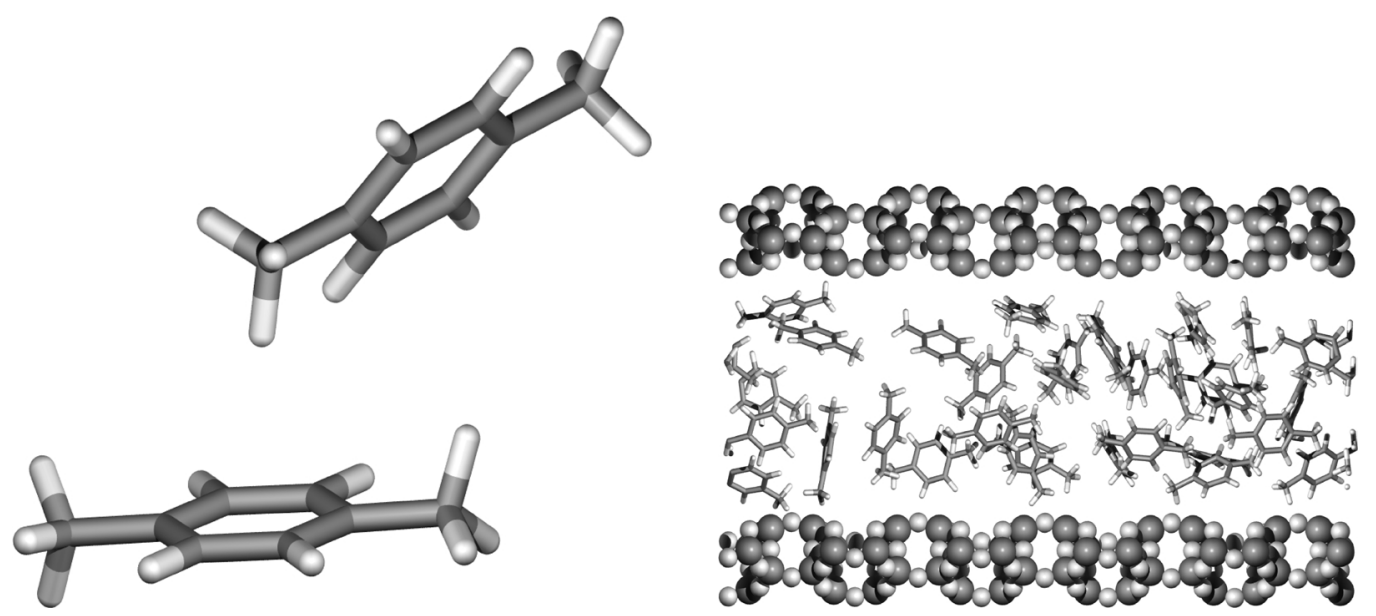

Fig. 8. Illustration of the configuration of the $p$-xylene dimer optimized at B3LYP/6-31G(d,p) level of theory (top) and a snapshot of $p$-xylene molecules in the center channel of the Si-MCM-41 supercell at loading of 64 molecules/supercell (bottom) after $100 \mathrm{ps}$ of MD equilibrium.

and the peak maximum increases strongly with loading. The increasing disorder is also indicated by the disappearance of the second peak, it being shifted toward smaller distances, thus filling-up of the minimum between the two.

\section{Conclusions}

A simple model of Si-MCM-41 has been constructed and used as a mesoporous host to study the adsorption and the diffusion of $p$-xylene. Reasonable agreement with the available experimental data is achieved with this new force field, providing confidence to use it in order to describe the adsorption and diffusion of aromatic hydrocarbons in this material. The findings shed some light on the factors involved in the evolution of the guest-guest and guest-host interactions with increasing loading. In this model, the heat of adsorption at zero loading is due primarily to short-range van der Waals interactions between the adsorbate and the walls, in keeping with chemical insight. With increasing loading, the cooperative effect of guest-guest interactions becomes more important

[1] C. T. Kresge, M. E. Leonowicz, W. J. Roth, J. C. Vatuli, and J. S. Beck, Nature 359, 710 (1992).

[2] J. S. Beck, J. C. Vartuli, W. J. Roth, M.E. Leonowicz, C. T. Kresge, K. D. Schmitt, C. T.-W. Chu, D. H. Olson, E. W. Sheppard, S. B. McCullen, J. B. Higgins, and J. L. Schlenkert, J. Am. Chem. Soc. 114, 10834 (1992). and is able to overcompensate the less favorable guesthost interactions, thus leading to an increase of the heat of adsorption. Due to the mutual steric hindrances and the confinement effect of the narrower pore MCM-41 used in this study, the variation of the molecular selfdiffusion coefficients with loading shows a trend of decreasing coefficients with increasing loading.

\section{Acknowledgements}

This work was supported in part by grants from the Thailand Research Fund (TRF Senior Research Scholar) to J.L., the Royal Golden Jubilee (RGJ) Ph.D. program and the Embassy of France in Thailand to T. N., as well as the Kasetsart University Research and Development Institute (KURDI), the Ministry of University Affairs under the Science and Technology Higher Education Development Project (MUAADB funds), and the National Nanotechnology Center (NANOTEC, Thailand). P.A.B. also thanks these sponsors for travel support and everybody at the LCAC of Kasetsart University for their hospitality. Computational resources from the Université Bordeaux 1 are also acknowledged.

[3] P.T. Tanev and T. J. Pinnavaia, Science 267, 865 (1995).

[4] R. Schmidt, D. Akporiaye, M. Stöcker, and O.H. Ellestad, Stud. Surf. Sci. Catal. 84, 61 (1994).

[5] R. B. Borade and A. Clearfield, Catal. Lett. 31, 267 (1995). 
[6] M. J. Girgis and Y. P. Tsao, Ind. Eng. Chem. Res. 35, 386 (1996).

[7] M. J. Climent, A. Corma, S. Iborra, M. C. Navarro, and J. Primo, J. Catal. 161, 783 (1996).

[8] N. Katada, T. Tsubouchi, M. Niwa, and Y. Murakami, Appl. Catal. A 124, 1 (1995).

[9] X. S. Zhao, G. Q. Lu, and G. J. Millar, Ind. Eng. Chem. Res. 35, 2075 (1996).

[10] B.P. Feuston and J. B. Higgins, J. Phys. Chem. 98, 4459 (1994).

[11] P.I. Ravikovitch, S. C. O. Domhnaill, A. V. Neimark, F. Schueth, and K.K. Unger, Langmuir 11, 4765 (1995).

[12] P. I. Ravikovitch, D. Wei, W. T. Chueh, G. L Haller, and A. V. Neimark, J. Phys. Chem. B 101, 3671 (1997).

[13] M. W. Maddox, J. P. Olivier, and K. E. Gubbins, Langmuir 13, 1737 (1997).

[14] K. Kleestorfer, H. Vinek, and A. Jentys, J. Mol. Catal. A 166, 53 (2001).

[15] D. Cao, Z. Shen, J. Chen, and X. Zhang, Microporous Mesoporous Mater. 67, 159 (2004).

[16] C. G. Sonwane and Q. Li, J. Phys. Chem. B 109, 5691 (2005).

[17] E. Armengol, M. L. Cano, A. Corma, H. García, and M. T. Navarro, J. Chem. Soc., Chem. Commun. 5, 519 (1995).

[18] C. Nguyen, C. G. Sonwane, S. K. Bhatia, and D. D. Do, Langmuir 14, 4950 (1998).

[19] V.R. Choudhary and K. Mantri, Microporous Mesoporous Mater. 40, 127 (2000).

[20] V. R. Choudhary and K. Mantri, Langmuir 16, 8024 (2000).

[21] J. Kärger, S. Vasenkov, and S. Auerbach, in: Handbook of Zeolite Science and Technology (Eds. S. M. Auerbach, K. A. Carrado, P. K. Dutta), Marcel Dekker, New York 2003, pp. $341-422$.

[22] A. Jentys, K. Kleestorfer, and H. Vinek, Microporous Mesoporous Mater. 27, 321 (1999).

[23] A. Chenite, Y. L. Page, and A. Sayari, Chem. Mater. 7, 1015 (1995).
[24] S. A. Walker and J. A. Zasadzinski, Mat. Res. Soc. Symp. Proc. 371, 93 (1995).

[25] V. Gusev, http://boba.bomm.ru/eng/mcm-41.html.

[26] G. Schrimpf, M. Schlenkrich, J. Brickmann, and P. A. Bopp, J. Phys. Chem. 96, 7404 (1992).

[27] T. Nanok, P.A. Bopp, and J. Limtrakul, work in progress.

[28] J. B. Nicholas, A. J. Hopfinger, F. R. Trouw, and L.E. Iton, J. Am. Chem. Soc. 113, 4792 (1991).

[29] V. A. Ermoshin, K. S. Smirnov, and D. Bougeard, Chem. Phys. 202, 53 (1996).

[30] W.D. Cornell, P. Cieplak, C.I. Bayly, I. R. Gould, K. M. Merz, D. M. Ferguson, D. C. Spellmeyer, T. Fox, J. W. Caldwell, and P. A. Kollman, Science 117, 5179 (1995).

[31] C. M. Breneman and K. B. Wiberg, J. Comput. Chem. 11, 361 (1990).

[32] V. R. Choudhary and K. Mantri, Langmuir 16, 7031, (2000).

[33] J.B. Nicholas and A.C. Hess, Science 116, 5428 (1994).

[34] W. Smith and T. R. Forester, The DLPOLY 2.0 User Manual. CCLRC, Daresbury Laboratory, Warrington, U. K. 1995.

[35] K. Shanmugapriya, M. Palanichamy, B. Arabindoo, and V. Murugesan, J. Catal. 224, 347 (2004).

[36] K. S. Smirnov and D. Bougeard, J. Phys. Chem. 97, 9434 (1993).

[37] A. Jentys, N.H. Pham, and H. Vinek, J. Chem. Soc. Faraday Trans. 92, 3287 (1996).

[38] R. Rungsirisakun, B. Jansang, P. Pantu, and J. Limtrakul, J. Mol. Struct. 733, 239 (2005).

[39] S.Z. Qiao, S. K. Bhatia, and D. Nicholson, Langmuir 20, 389 (2004).

[40] J. H. Kim and S. H. Lee, Bull. Korean Chem. Soc. 23, 447 (2002).

[41] E. Gedat, A. Schreiber, G. H. Findenegg, I. Shenderovich, H.-H. Limbach, and G. Buntkowsky, Magn. Reson. Chem. 39, S149 (2001). 